\title{
Cluster observations of mid-latitude hiss near the plasmapause
}

\author{
A. Masson ${ }^{1}$, U. S. Inan ${ }^{2}$, H. Laakso ${ }^{1}$, O. Santolík ${ }^{3}$, and P. Décréau ${ }^{4}$ \\ ${ }^{1}$ RSSD of ESA, ESTEC, Noordwijk, 2201AZ, The Netherlands \\ ${ }^{2}$ STAR Laboratory, Stanford University, Stanford, CA 94305, USA \\ ${ }^{3}$ Charles University, Prague, Czech Republic \\ ${ }^{4}$ LPCE/CNRS and Université d'Orléans, Orléans, 45071, France
}

Received: 1 October 2003 - Revised: 24 May 2004 - Accepted: 24 June 2004 - Published: 14 July 2004

Part of Special Issue "Spatio-temporal analysis and multipoint measurements in space"

\begin{abstract}
In the vicinity of the plasmapause, around the geomagnetic equator, the four Cluster satellites often observe banded hiss-like electromagnetic emissions (BHE); below the electron gyrofrequency but above the lower hybrid resonance, from $2 \mathrm{kHz}$ to $10 \mathrm{kHz}$. We show that below $4 \mathrm{kHz}$, these waves propagate in the whistler mode. Using the first year of scientific operations of WHISPER, STAFF and WBD wave experiments on Cluster, we have identified the following properties of the BHE waves: (i) their location is strongly correlated with the position of the plasmapause, (ii) no MLT dependence has been found, (iii) their spectral width is generally 1 to $2 \mathrm{kHz}$, and (iv) the central frequency of their emission band varies from $2 \mathrm{kHz}$ to $10 \mathrm{kHz}$. All these features suggest that BHE are in fact mid-latitude hiss emissions (MLH). Moreover, the central frequency was found to be correlated with the $K_{p}$ index. This suggests either that these banded emissions are generated in a given $f / f_{c e}$ range, or that there is a $K_{p}$ dependent Doppler shift between the satellites and a possible moving source of the MLH.
\end{abstract}

Key words. Magnetospheric physics (plasmasphere; plasmapause; whistler mode waves)

\section{Introduction}

The plasmasphere is an inner magnetospheric region, located above the ionosphere. Its shape is usually compared to a torus around the Earth, which bulges out to the dusk side. At the equator, it typically extends out to distances of $\approx 4-5$ Earth radii. Its density is high and its temperature low $\left(>100 \mathrm{~cm}^{-3}, \sim 1 \mathrm{eV}\right)$ compared to the outer tenuous and warm magnetospheric plasma (a few e-/cc, $100-1000 \mathrm{eV}$ ). Its outer boundary, the plasmapause, separates the plasmasphere from the rest of the magnetosphere.

Correspondence to: A. Masson

(amasson@rssd.esa.int)
The plasmapause was observed by Carpenter (1963) after analyzing whistlers, measured by ground based Very Low Frequency (VLF, 3-30 kHz) receivers. Whistlers are VLF electromagnetic waves produced by lightning, which have a typical whistling tone when turned to audio. They propagate through the magnetosphere in a mode of propagation called the whistler mode (Stix, 1992).

Whistlers are not the only waves that propagate in the whistler mode, e.g. auroral hiss, chorus, mid-latitude hiss, narrow-band plasmapause hiss, plasmaspheric hiss (Sazhin, 1993; Ondoh, 1993; Sonwalkar, 1995). All these waves are observed at frequencies below the electron gyrofrequency, $f_{c e}$, or the plasma frequency, $F_{p}$, whichever is lower; typically in the VLF and the Extremely Low Frequency (ELF) ranges. In this paper, ELF covers the following frequency range: $3 \mathrm{~Hz}-3 \mathrm{kHz}$.

Through wave-particle interactions, whistler mode waves can perturb the underlying ionosphere by scattering radiation belt electrons from their orbits (Helliwell et al., 1973; Burgess and Inan, 1993). The ionospheric perturbations produced, include X-rays, optical emissions, and density enhancements (e.g. Rosenberg et al., 1971; Helliwell and Mende, 1980; Paschmann et al., 2003).

The Cluster space fleet perigee passes $(L \sim 4)$ provide excellent opportunities to study whistler mode waves near the equatorial plane, which is indeed the source location of some whistler mode waves (e.g. chorus). Moreover, in the vicinity of the plasmapause, the equatorial region appears to be a favored location for amplification of (nearly) constant frequency whistler mode signals (Helliwell, 1988; Lemaire and Gringauz, 1998).

Moullard et al. (2002) reported an unidentified $3-6 \mathrm{kHz}$ whistler mode emission, observed by all Cluster spacecraft near perigee, on 5 June 2001. This event was measured inside and just outside the plasmasphere ( $\sim 1 \mathrm{~h}$ duration). This emission band appeared anti-correlated with large density enhancements, suggesting trough ducting. The authors argued that these waves could be generated near or at the 
plasmapause, propagating back and forth with respect to the geomagnetic equator. Data from one Cluster satellite were displayed and no polarization evidence was presented.

A similar band-limited emission was observed by all Cluster spacecraft during $\sim 30 \mathrm{~min}$, on 7 October 2001 (see Fig. 1). Like mid-latitude hiss, its frequency range is included between $\sim 2$ and $10 \mathrm{kHz}$ (Sonwalkar, 1995). Figure 2 displays fine details of this $5-6 \mathrm{kHz}$ emission during $30 \mathrm{~s}$. Rising tones, possibly whistlers, are embedded in the midst of the band (see white arrows in Fig. 2). This observational fact is also in common with mid-latitude hiss (Sonwalkar, 1995).

But these few similarities are not enough to qualify these two band-limited emissions as mid-latitude hiss (for a review, see Hayakawa and Sazhin, 1992). A survey needs to be conducted to check their geophysical properties. Meanwhile, these emissions will be called banded hiss-like emission (BHE); banded hiss is a VLF hiss, banded in frequency, which has long been observed on the ground in middle latitudes (e.g. Morgan, 1979).

We have found twenty-seven cases of BHE over the first 14 months of the Cluster mission. In this paper, we present, for the first time, some geophysical properties of the BHE, by attempting to answer the following questions: (i) what are the common features between these 27 cases?, (ii) what can we learn about the BHE source location through multipoint measurements?, (iii) what are the propagation features of the BHE?, (iv) is there any magnetic latitude or magnetic local time dependence?, (v) why have these waves not been observed and studied by previous satellites like DE-1 or Polar?, (vi) is it always the same frequency band? If not, is there any correlation with the geomagnetic activity? And finally, can we qualify the BHE as mid-latitude hiss?

\section{Cluster instruments and data products used}

The four Cluster spacecraft (thereafter called C1, C2, C3 and C4) were successfully launched in pairs in July and August 2000. After six months of maneuvers and commissioning, the scientific mission started on 1 February 2001. The wave sensors on each satellite consist of a boom-mounted threeaxis search coil magnetometer and four electric probes at the ends of four $42.5 \mathrm{~m}$ long booms in the spin plane (Escoubet et al., 1997a). Electric field spectrograms from 2 to $80 \mathrm{kHz}$ are provided by the Waves of HIgh frequency and Sounder for Probing of Electron density by Relaxation (WHISPER) experiment (Décréau et al., 1997). Below $4 \mathrm{kHz}$, electric and magnetic spectra are provided by the Spatio-Temporal Analysis of Field Fluctuations (STAFF) experiment (Cornilleau-Wehrlin et al., 1997). The WideBanD (WBD) experiment provides very high-rate digital samples of electric and magnetic waveforms in three possible frequency bands, $25 \mathrm{~Hz}-9.5 \mathrm{kHz}, 50 \mathrm{~Hz}-19 \mathrm{kHz}$, and $1 \mathrm{kHz}-$ $77 \mathrm{kHz}$ (Gurnett et al., 1997).

The data used for this survey are primarily WHISPER spectrograms measured from February 2001 to March 2002, and WBD high time resolution waveforms, turned to spectrograms. STAFF data analysis was performed when the BHE were observed below $4 \mathrm{kHz}$.

\section{Properties and common features of the BHE}

Twenty-seven BHE cases have been found over the first 14 months of scientific operations of Cluster. They are all listed in the Appendix.

Each time, their spectral width is of the order of 1 to $2 \mathrm{kHz}$. Their frequency bandwidth is always between $2 \mathrm{kHz}$ and $10 \mathrm{kHz}$, in between the lower hybrid resonance $f_{l h}(\sim 300$ $400 \mathrm{~Hz})$ and $f_{c e}(\sim 12 \mathrm{kHz})$. They last from a few minutes to an hour, always corresponding to middle invariant latitudes (i.e. included in the $50^{\circ}-67^{\circ}$ range).

Thanks to the multipoint measurements of Cluster, some features of the BHE source may be deduced (Sect. 3.1). The electromagnetic nature of the BHE and its propagation characteristics are then presented through the analysis of a case study (Sect. 3.2). Moreover, as the Cluster mission covers all MLT sectors over one year, the question of a possible MLT dependence of the BHE is addressed in Sect. 3.3. All these properties, together with the analysis of an apparent magnetic latitude confinement, will help to understand why Cluster often observes these waves (Sect. 3.4). The geomagnetic activity dependence of the central frequency of the BHE is investigated in Sect. 3.5. Whether or not qualifying these BHE as mid-latitude hiss is discussed in Sect. 3.6.

\subsection{Features of the BHE source by multipoint analysis}

Redundant features of the BHE source will now be illustrated on two significant cases: 7 and 10 October 2001. As a reminder, the light blue curve (going on Fig. 1 from 30 to $80 \mathrm{kHz}$ and then from 80 to $10 \mathrm{kHz}$ ) represents natural emissions whose lower cutoff corresponds to $F_{p}$. This cutoff enables one to follow the electron density $\left(N_{e}\right)$, with $F_{p}^{2}(\mathrm{kHz})$ $\sim 81 N_{e}\left(\mathrm{~cm}^{-3}\right)$.

On 7 October 2001 (Fig. 1), a BHE $\sim 5 \mathrm{kHz}$ is observed simultaneously on C1, C2 and C4 (16:25-17:04 UT). As one can see in the top panel of Fig. 3, the lower the altitude, the more intense the BHE: $\mathrm{C} 4>\mathrm{C} 2>\mathrm{C} 1$. Forty-five minutes later, when $\mathrm{C} 3$ arrives in the plasmapause, following nearly the same orbit as $\mathrm{C} 2$, the intensity of the BHE has strengthened. This fact has at least three possible explanations. Either the BHE source is moving towards C3, or the intensity of the source has increased, or a mix of these two possibilities.

In more than $80 \%$ of the cases selected, the BHE is observed on each of the four Cluster satellites. Within the five remaining cases, the BHE is only observed on three, two or one spacecraft.

Such an event occurred on 10 October 2001 (see Fig. 4), only three days after the previous case. Between these two events, the geomagnetic activity has significantly increased. The bottom panel of Fig. 3 illustrates this increase by presenting the evolution of the $K_{p}$ and the $A_{p}$ index. During 

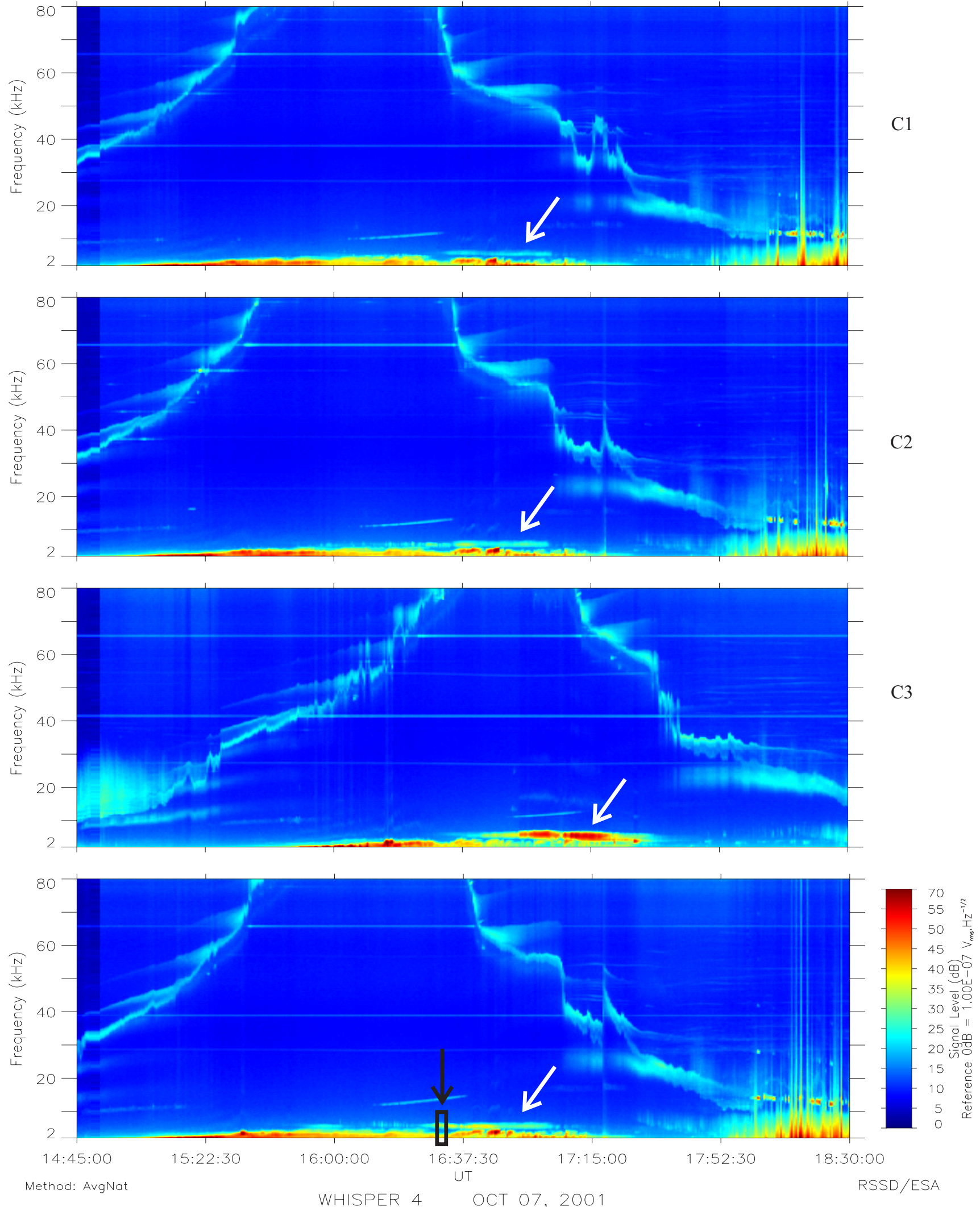

Fig. 1. From top to bottom, WHISPER spectrograms measured by C1 to C4 on 7 October 2001, from 14:45 UT to 18:30 UT. Note the presence of a banded hiss-like emission from 16:25 UT to 17:04 UT, pointed out by white arrows, which intensifies with altitude: $\mathrm{C} 4>\mathrm{C} 2>\mathrm{C} 1$ (see right panel of Fig. 3) and with time $\mathrm{C} 3 \gg \mathrm{C} 4>\mathrm{C} 2>\mathrm{C} 1$. In the last panel, the black box, pointed out by a black arrow, symbolizes the time period and the frequency range of the enlargement displayed in Fig. 2. 


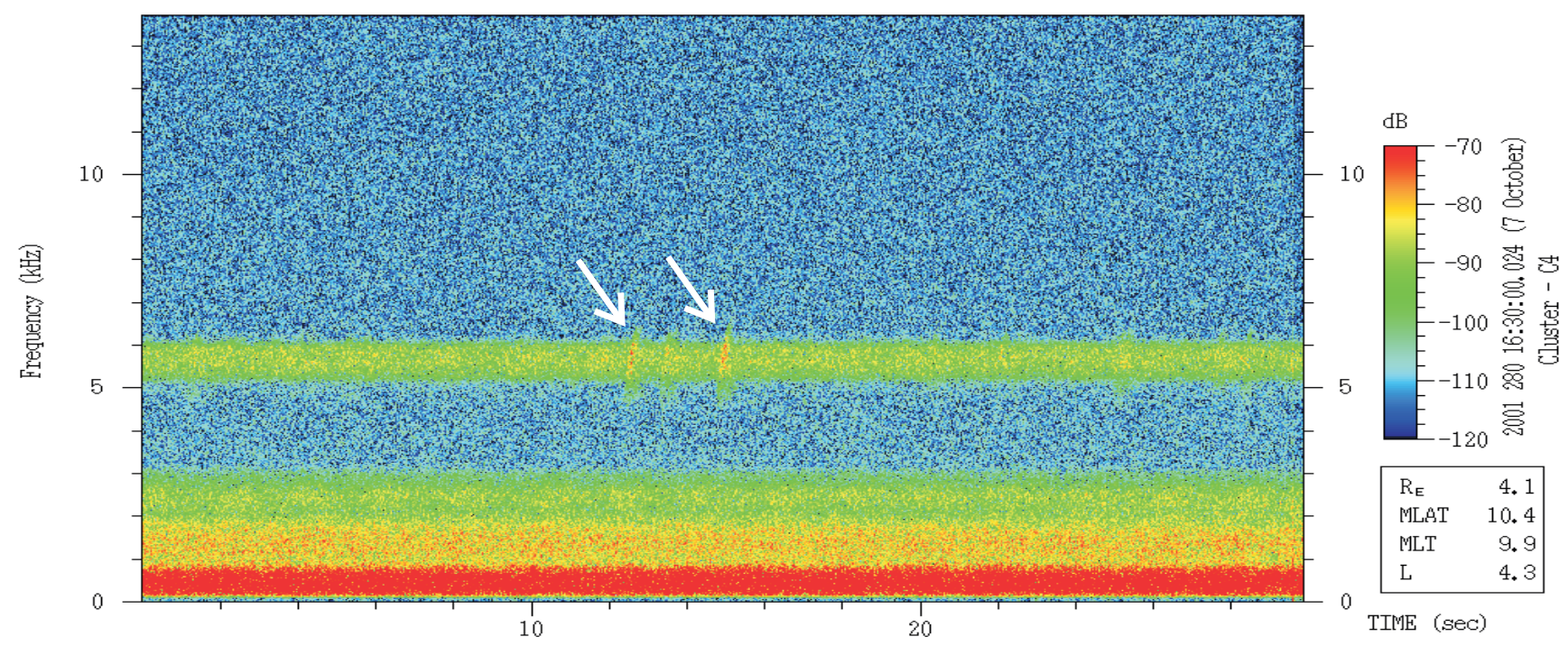

Fig. 2. High time resolution WBD electric field spectrogram measured by C4 on 7 October 2001, from 16:00:00.024 UT to 16:00:30.024 UT. The banded hiss-like emission (BHE) is observed just above $5 \mathrm{kHz}$. Two rising tones, possibly whistlers, are embedded in this BHE. They are pointed out by white arrows around 16:00:12 UT and 16:00:15 UT.
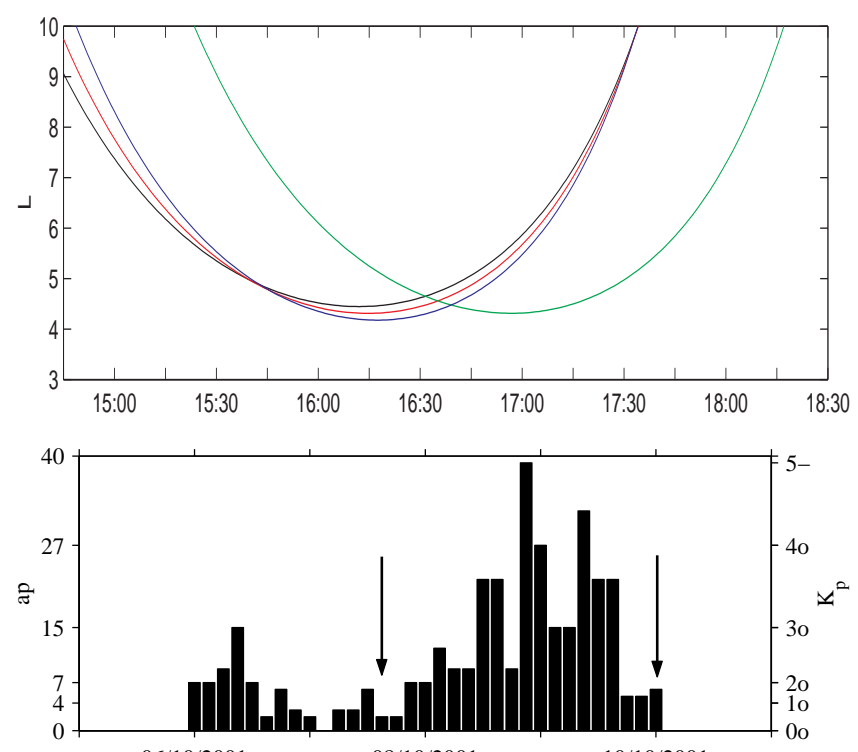

Fig. 3. Top panel: orbit of the 4 Cluster satellites versus time on 7 October 2001 (1: black; 2: red; 3: green; 4: blue); bottom panel: evolution of the $A_{p}$ and the $K_{p}$ indices during 6-10 October 2001 period, the arrows point at the time of the Cluster perigee passes.

this period, the plasmasphere has been thus compressed but the Cluster perigee still remains near $4 R_{E}$. Therefore, while on 7 October the electron density exceeded $80 \mathrm{~cm}^{-3}$ for almost an hour (e.g. between 15:30 and 16:30 UT on C1), three days later, the electron density does not exceed $45 \mathrm{~cm}^{-3}$ $\left(F_{p}<60 \mathrm{kHz}\right)$ on $\mathrm{C} 2, \mathrm{C} 3$ and $\mathrm{C} 4$, and not even $30 \mathrm{~cm}^{-3}$ $\left(F_{p}<50 \mathrm{kHz}\right)$ on $\mathrm{C} 1$ (see Fig. 4$)$.

On 10 October 2001, the BHE is clearly seen around $8 \mathrm{kHz}$ on C4, between 01:29 and 01:41 UT. The same emission is barely visible on $\mathrm{C} 2$ and $\mathrm{C} 3$. No presence of this emission can be seen on $\mathrm{C} 1$. This means that the Cluster spacefleet has to enter deep enough into the plasmapause to observe the BHE.

\subsection{STAFF-SA analysis}

Among the 27 BHE cases selected, eight of them have their frequency band lying, at least partially, below $4 \mathrm{kHz}$ (STAFF upper frequency cutoff). All these STAFF cases are listed in the Appendix.

An example of such a case is presented in Fig. 5, measured by $\mathrm{C} 1$ on 5 June 2001. This figure shows $2 \mathrm{~h}$ of WHISPER (panel a) and STAFF (panels $b$ to g) data, recorded during a perigee pass. The spacecraft is located in the dusk sector and moves along its orbit from the Southern to the Northern Hemisphere. The geomagnetic equatorial plane is crossed between 21:46 and 21:47 UT (zero crossing of the C1 Solar Magnetic latitude). Here, the BHE is banded inside the 3$6 \mathrm{kHz}$ range, observed from 21:20 to 22:20 UT (see panel a). The other panels of Fig. 5 display STAFF data and related data products from $60 \mathrm{~Hz}$ to $4 \mathrm{kHz}$, in logarithmic scale: (b) electric field spectrogram, (c) magnetic field spectrogram, (d) polarization planarity $F$, (e) parallel component of the Poynting vector, normalized by its standard variation, (f) polarization ellipticity $L$ and (g) angle between the wave normal direction and the DC magnetic field. The calculations of the last four quantities are based on methods detailed in the following papers and references therein (Santolík et al., 2001, 2003).

Before describing the BHE propagation characteristics above $2 \mathrm{kHz}$, let us first describe the most obvious feature of panels (b) to $(\mathrm{g})$ : electromagnetic waves below $f_{l h}(\sim 400 \mathrm{~Hz})$ but above the proton gyrofrequency $f_{H+}$ $(\sim 10 \mathrm{~Hz})$, from 21:20 to 22:06 UT. During this time period 

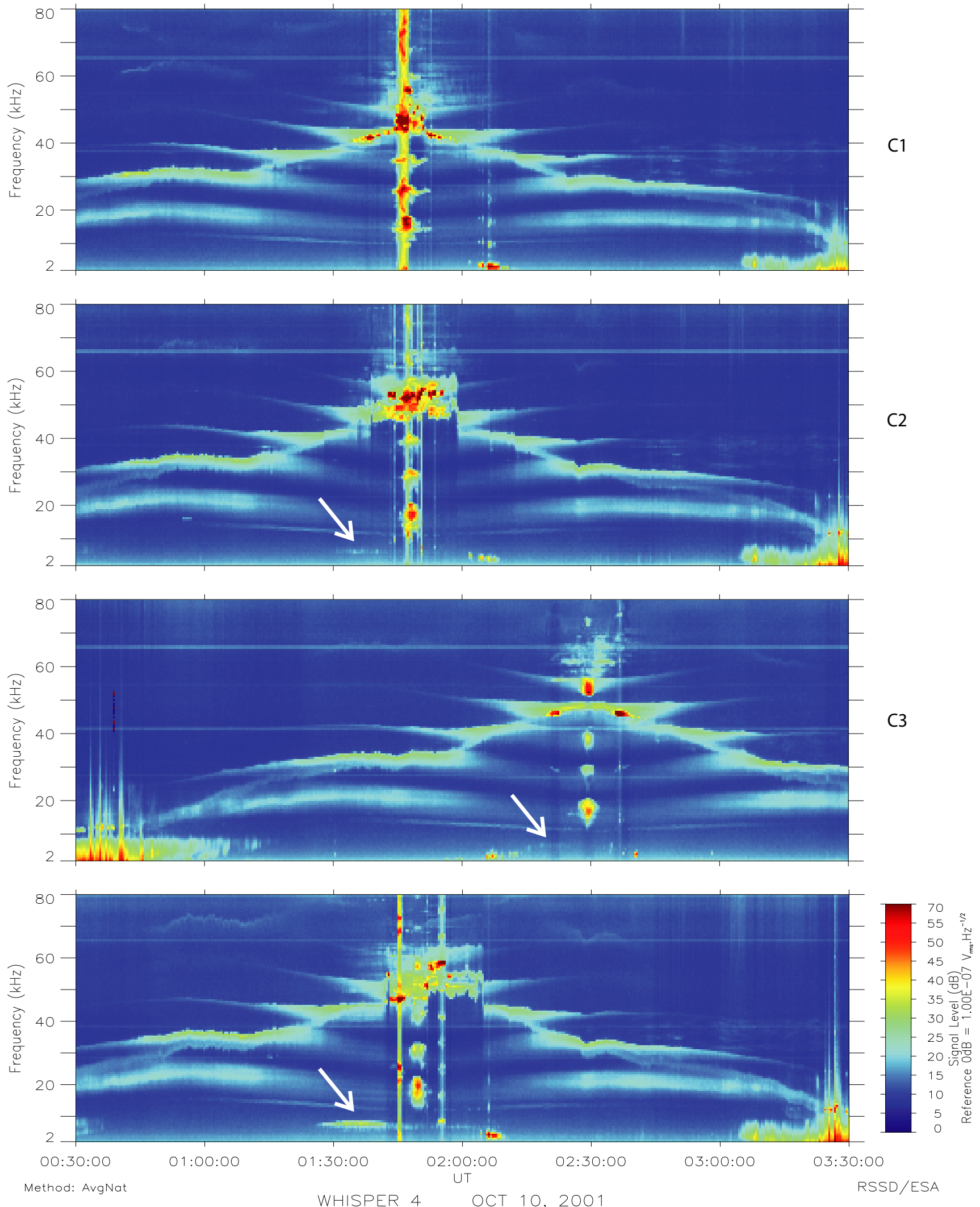

Fig. 4. WHISPER spectrograms measured by the four Cluster spacecraft on 10 October 2001, 00:30-03:30 UT. Note the presence of a short duration banded hiss-like emission, just after 01:30 on C4 and C2 (far less intense), and barely distinguishable 45 min later on C3. 
(a)
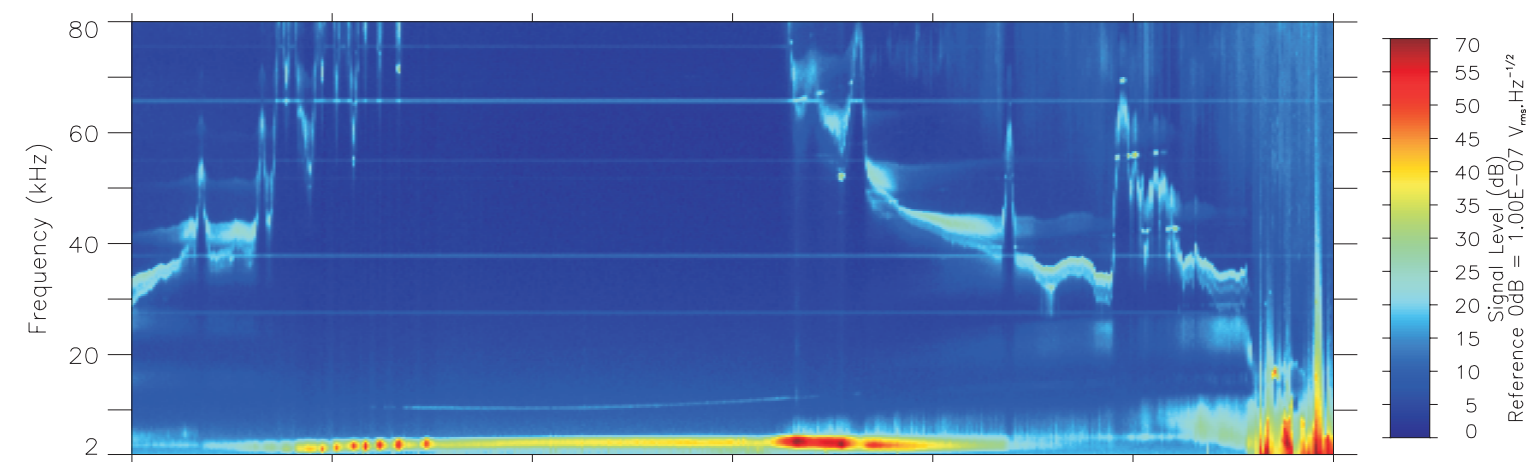

(b)

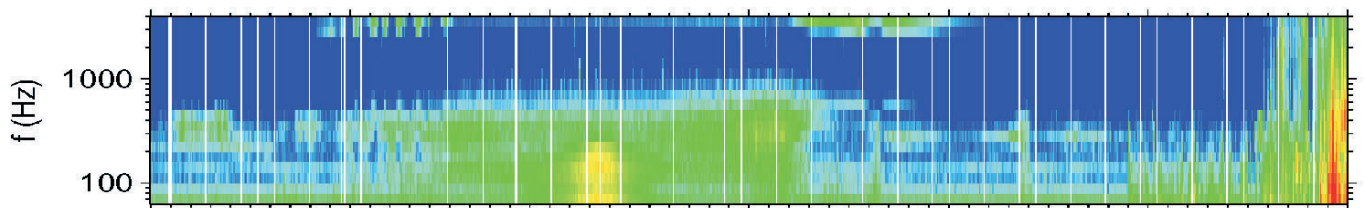

(c)
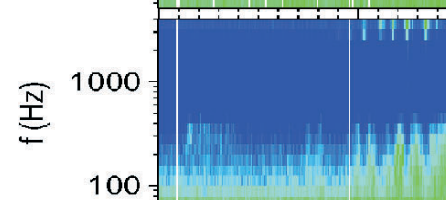

$$
\text { i }
$$

$$
\text { (d) } \stackrel{\mathbb{x}}{\underline{x}}_{1000}^{100}
$$
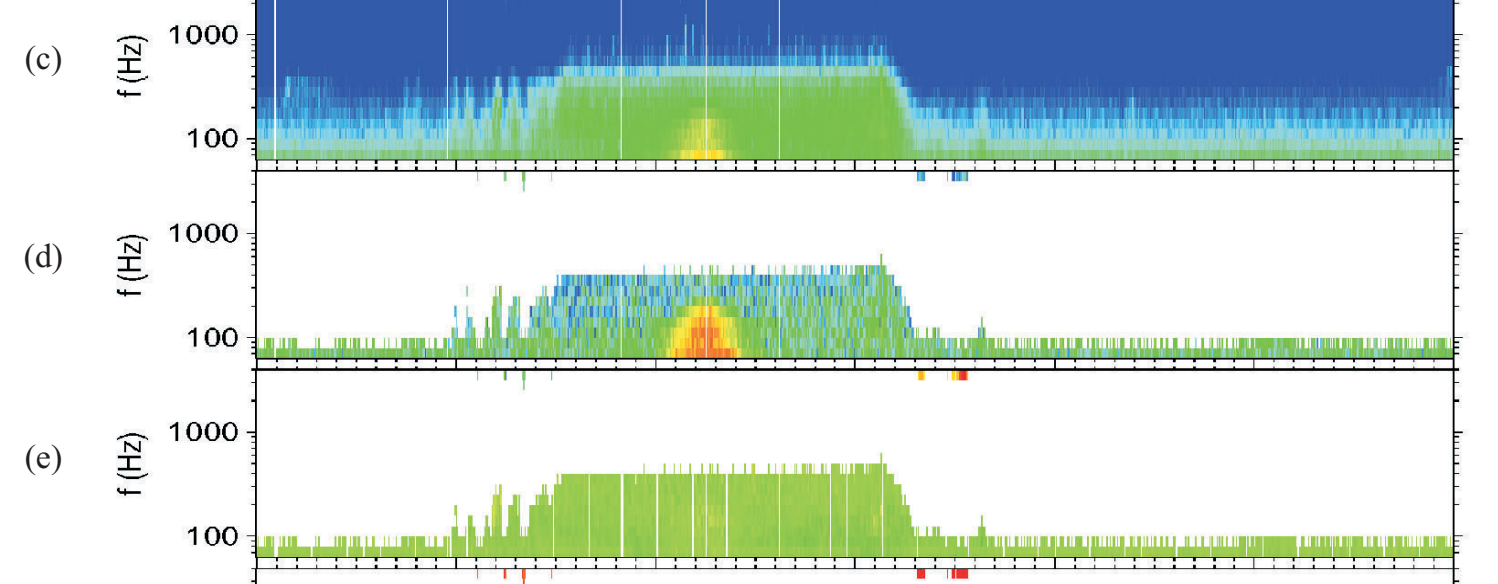

(f)
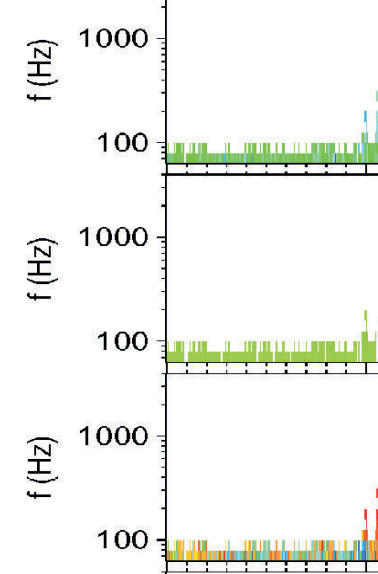
In a clear distinction from the equatorial noise, region 2 waves are characterized by low $F$ values (panel d) and highly positive $L$ values (panel $\mathrm{f}$ ). This latter point implies that region 2 waves are elliptically polarized, and propagate in the right-hand polarized mode, like ELF hiss (Santolík and Parrot, 2000; see Hayakawa and Sazhin, 1992 for a review). Let us now come back to the description of the BHE propagation characteristics.

Banded hiss-like emission is a far less visible feature of panels (b) to (g). Indeed, for each of these panels, only the values from 2 to $4 \mathrm{kHz}$ are related to the $\mathrm{BHE}$. This frequency range is located near the upper edge of each record. For this event, BHE is observed on each side of the geomagnetic equator, crossed by $\mathrm{C} 1$ around 21:45 UT.

Panel (c) shows that the BHE is an electromagnetic emission from $2 \mathrm{kHz}$ to $4 \mathrm{kHz}$. The scale on this figure has been chosen so that the dark blue color represents any value below $5.10^{-9} \mathrm{nT}^{2} \cdot \mathrm{Hz}^{-1}$. Due to this scale, only the most intense magnetic component shows up.

In panel (d), low values of the polarization planarity are found on both sides of the equator, like for plasmaspheric hiss.

Panel (e) displays the parallel component of the Poynting vector, normalized by its standard variation. North of the geomagnetic equator, the value of this component is high (red values), corresponding to the propagation of these waves towards north. South but close to the equator, this quantity is blue, corresponding to a propagation towards south. This propagation behavior is similar to chorus emissions. Further south, this quantity becomes green, meaning propagation in both directions.

Panel (f) shows that on both sides of the geomagnetic equator the ellipticity of the polarization is close to +1 , meaning that the waves are right-hand elliptically (nearly circularly) polarized.

The last panel $(\mathrm{g})$ shows that the angle between the wave normal direction of these waves and the DC magnetic field is of the order of $30^{\circ}$. This angle can increase to $60^{\circ}$ when emissions are observed further away from the equator.

\subsection{MLT dependence}

The Cluster orbit was chosen with a perigee at $4 R_{E}$, an apogee at $19.6 R_{E}$, an inclination of $90^{\circ}$ and a line of apsides around the ecliptic plane (Escoubet et al., 1997b). The orbital period is $57 \mathrm{~h}$, i.e. $\sim 2.5$ days. In December 2000 , four months after launch, the spacefleet was orbiting in the dawndusk meridian with perigee in the dawn sector. Then, as the Earth rotates around the Sun, the perigee was around midnight after 3 months (March 2001), at dusk after 6 months (June 2001), around noon after 9 months (September 2001) and then again in the dawn sector one year later. As a consequence, by analyzing a specific phenomenon at perigee over a full year of Cluster data, one is able to study its presence and its geophysical properties at all magnetic local times.

As one can notice in Fig. 6 (left panel), the BHE tend to occur at all MLT sectors: seven cases found in the 21:00-
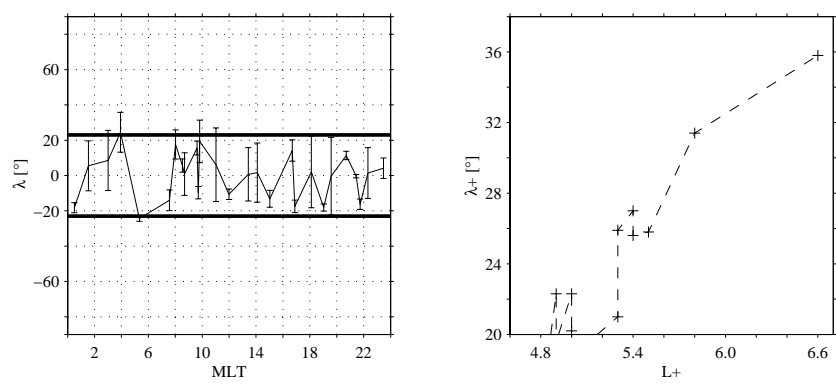

Fig. 6. Left panel: magnetic latitude ranges vs. MLT; right panel: magnetic latitude upper absolute value vs. L-shell.

03:00 MLT sector, six in the 03-09 sector, seven in the 09-15 sector and seven in the 15-21 sector. The absence of MLT dependence is also a typical feature of mid-latitude hiss (Sonwalkar, 1995).

This lack of MLT dependence has some consequences in the bulge region. As a reminder, the plasmasphere encompasses a bulge region, which extends out to greater distances most of the time in the 19:00-22:00 MLT sector (Higel et $\mathrm{Wu}, 1984)$. Meanwhile, the four Cluster spacecraft always have their perigee near $L=4$. We found six cases in this MLT sector but always with high $K_{p}$ values during the preceding $24 \mathrm{~h}$ (typical values included in the $3+, 6+$ range). For such geomagnetic activity, the bulge region of the plasmasphere turns noonward (Carpenter et al., 1993). Therefore, the cases found in this MLT sector could be explained by the fact that the BHE is strongly linked with the plasmapause itself.

\subsection{Magnetic latitude dependence}

In the vicinity of the plasmapause, the geomagnetic equator is known to be a region of damping and amplification of waves due to the presence of anisotropic distributions of 1$30 \mathrm{keV}$ electrons. Unfortunately, before January 2003, the Cluster electron instrument PEACE (Johnstone et al., 1997) was always turned off below $L=6$, in order to avoid any damage from the radiation belts. Therefore, for this study, we cannot investigate a possible correlation between the BHE and electron characteristics.

The Solar Magnetic latitude $(\lambda)$ ranges versus MLT relationship for all BHE events was studied to check whether these waves are only observed near the equator. For each BHE event, the upper and lower $\lambda$ values have been deduced on each spacecraft, using the start and end time of each BHE. When the waves were observed on more than one spacecraft, the minimum and maximum values among the different spacecraft were chosen to establish the magnetic latitude range of the event.

In more than $75 \%$ of all the events, the $\lambda$ ranges fall into the $\left[-23^{\circ}, 23^{\circ}\right]$ range (see left panel of Fig. 6). For these cases, the related maximum $L$-shell value, $L_{+}$, was always equal or below 5.3. For less than $25 \%$ of the events, the magnetic latitude upper value $\left(\lambda_{+}\right)$of each event ranged from $25^{\circ}$ to $36^{\circ}$ (in absolute value). 


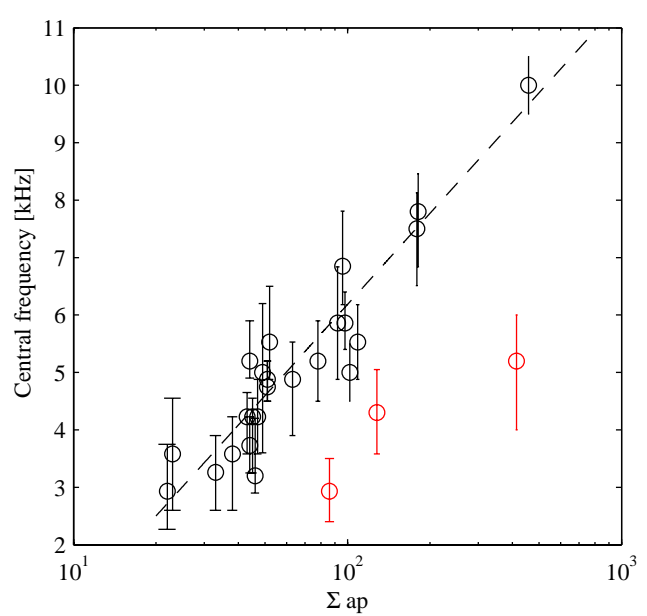

Fig. 7. Central frequency and spectral width of the BHE vs. the preceding $24 \mathrm{~h}$ sum of $A_{p}$ indices.

The $\lambda_{+}$values are plotted against their corresponding $L_{+}$ values in the right panel of Fig. 6. Clearly, for increasing $L_{+}$, $\lambda_{+}$increases. This result is not surprising considering the strong dependence of the plasmapause position with regards to the geomagnetic activity, using $K_{p}$ as an indicator (Carpenter and Anderson, 1992). Indeed, all these events correspond to periods of quiet geomagnetic activity $\left(K_{p}\right.$ goes from $2^{\circ}$ to $\mathrm{O}^{\circ}$ as $\lambda_{+}$goes from $25^{\circ}$ to $36^{\circ}$ ), for hours before each Cluster plasmaspheric crossing. During such periods, the plasmasphere expands and is filled with ionospheric plasma, but at the same time the Cluster spacefleet still crosses the geomagnetic equator around $4 R_{E}$. These higher $\lambda_{+}$values might be just the consequence of both the plasmasphere expansion and the Cluster orbit.

The orbit of the spacefleet seems to indeed play a crucial role in the way we are observing the BHE on Cluster.

The $\left[-23^{\circ}, 23^{\circ}\right]$ apparent confinement of the BHE seems rather due to the Cluster orbit, reminding us that their mode of propagation should allow these waves to propagate along the field lines and not being confined.

Other satellites like DE-1 or Polar did not observe the BHE also because of their orbit, as none of these satellites were crossing the geomagnetic equator around $4 R_{E}$ and skimming the plasmapause for about one hour.

\subsection{Geomagnetic activity dependence}

The central frequency and the spectral width values of all the BHE have been gathered and compared to the geomagnetic activity. The proxy chosen for the geomagnetic activity is the sum of $K_{p}$ values (turned to $A_{p}$ ) over the preceding $24 \mathrm{~h}$. Figure 7 displays, for each event, the central frequency (circles) and the spectral width (error bar) with respect to the sum of $A_{p}$ over the preceding $24 \mathrm{~h}$. It has to be mentioned that in the case of periods centered at 09:00, 12:00, and 15:00 MLT, respectively, the values for one, two or three immediately preceding 3-h periods were ignored to account for observed delays in the response of the dayside radius to enhanced convection activity (Lemaire and Gringauz, 1998, and references therein). As one can see, the frequency band of most of the events lies in the $3 \mathrm{kHz}-6 \mathrm{kHz}$ for range moderate geomagnetic activity. Nevertheless, it seems that the higher the geomagnetic activity, the higher the central frequency of the BHE. A best fit has been derived from these events relating the central frequency value to the geomagnetic activity: $\mathrm{F}_{o}[\mathrm{kHz}]=2.3 * \ln \left(\Sigma A_{p}\right)-4.4$. Note that the values of three events measured around 21:00 MLT have not been taken into account in this calculation.

There are two possible explanations for the linear relationship in Fig. 7. The first one is to assume that these waves are generated in the vicinity of the plasmapause, near the geomagnetic equator, in a given $f / f_{c e}$ frequency bandwidth. Now let us remember that the equatorial $f_{c e}$ value near the plasmapause is $1 / L^{3}$ dependent. When the geomagnetic activity is high, the plasmapause will be compressed and its location will be closer to the Earth, hence its L-shell position will decrease. In this case $f_{c e}$ will increase, and hence the central frequency of the BHE will increase too, with respect to our assumption.

Another possible explanation is to assume the presence of a moving source located within the plasmapause, whose central frequency seems to be around 4 to $5 \mathrm{kHz}$. The central frequency drift would then be explained by a Doppler shift.

\section{Discussion}

Our study shows that, in the immediate vicinity of the plasmapause, the four Cluster spacecraft regularly observe a VLF emission with the following characteristics:

- between $2 \mathrm{kHz}$ and $10 \mathrm{kHz}$, always below the electron gyrofrequency and the plasma frequency but above the lower hybrid resonance,

- 1-2 kHz wide,

- banded in frequency,

- electromagnetic,

- right-hand elliptically polarized,

- escaping from the equator,

- structure less,

- rising tones sometimes embedded within,

- no MLT dependence found,

- lasting from a few minutes to an hour, always corresponding to invariant latitude from $50^{\circ}$ to $67^{\circ}$.

According to this list of properties, the BHE has similarities with a narrow-band plasmapause hiss observed by the ISIS 1 and ISIS 2 satellites (Ondoh et al., 1980, 1981; Ondoh, 1993). However, the central frequency of this banded hiss was so close to $5 \mathrm{kHz}$ that it was first called narrow-band 
5-kHz hiss (Ondoh et al., 1980, 1981). In Ondoh (1993), close similarity of this narrow-band hiss observed by the ISIS satellites with that observed on the ground (Syowa station, Antarctica) suggest that this hiss is electromagnetic, propagating in the whistler mode. The central frequency of this narrow-band hiss remains around $5 \mathrm{kHz}$ over the $56^{\circ}-65^{\circ}$ invariant latitude range, corresponding to the plasmapause location. The hiss was therefore renamed narrow-band plasmapause hiss.

On page 641 of (Ondoh, 1993), it is written: "All f-t spectra of narrow band hiss observed around $5 \mathrm{kHz}$ by the ISIS satellites show no lower frequency cutoff". This feature is a clear difference between this emission and the BHE, as illustrated in Fig. 2. Another difference is the maximum occurrence of the narrow-band plasmapause hiss in the late evening sector (quiet and moderate conditions) and in the midnight sector (disturbed). As presented in our study, no MLT dependence is found, even though our study is based on 27 cases only.

In Hayakawa and Sazhin (1992), no difference is made between narrow-band $5-\mathrm{kHz}$ hiss and mid-latitude hiss. The latter may be indeed considered as a more general denomination. In other words, narrow-band plasmapause hiss might be seen as a subset of a more general VLF hiss emission: mid-latitude hiss.

As reviewed in Sonwalkar (1995), at ground stations, midlatitude hiss is observed at invariant latitudes of about $20^{\circ}$ to $70^{\circ}$ and peaks near latitudes $55^{\circ}$ to $65^{\circ}$ (Helliwell, 1965; Hayakawa and Sazhin, 1992). Like on 5 June 2001 (Fig. 5), it usually appears as a band limited white noise in a 3 to $8 \mathrm{kHz}$ band, lasting for an hour or so, with a relatively smooth rise and fall. Simultaneously, whistlers are rather frequently observed in the same frequency band. This could explain the presence of rising tones, possibly whistlers, in Fig. 2. Among the 27 cases selected, their presence is also rather frequent.

The possible interaction between the observed rising tones and the BHE requires further investigation. Dowden (1971) suggested that whistlers could play a role in the generation of mid-latitude hiss. Another type of interaction is the effect of noise suppression by whistlers, frequently observed near $L=4$ (Gail and Carpenter, 1984). The propagation of noise bands is also observed just outside the plasmapause near $L=4$ to a ground station, in the early stages of a weak magnetic storm (Carpenter, 1978). A future survey is foreseen on the interaction between whistlers and the BHE observed on Cluster, with a more focused High-resolution WBD data set.

The hiss-type ELF emission, described in Sect. 3.2 as region 2 waves, is another issue for future investigation. This hiss-type ELF emission, most likely plasmaspheric hiss, is reviewed in Hayakawa and Sazhin (1992). The main energy of these emissions is observed between $100 \mathrm{~Hz}$ and $1 \mathrm{kHz}$ but can extend to a few $\mathrm{kHz}$. As mentioned in Hayakawa and Sazhin (1992 and references therein), it seems possible that the high frequency part of the plasmaspheric hiss may sometimes result from the influence of mid-latitude hiss. Further investigation based on a large dataset of Cluster data might help providing some answers about this possible influence.
A significant number of satellites have observed midlatitude hiss, as detailed in (Hayakawa and Sazhin, 1992). An additional satellite might be added to the list of satellites provided in this review paper: Ogo 6. For example, Morgan (1979) presents mid-latitude hiss measurements centred at about $7 \mathrm{kHz}$ and $2 \mathrm{kHz}$ wide. However, he calls this emission banded hiss.

Based on all these previous observations and the present study, it seems most likely that banded hiss-like emission is in fact mid-latitude hiss.

\section{Conclusion}

In Moullard et al. (2002), a case study reported an unidentified $3-6 \mathrm{kHz}$ whistler mode emission, observed by Cluster near the plasmapause.

Our study presents observational evidence that this VLF emission is most likely mid-latitude hiss (MLH). Our survey indeed presents several geophysical properties in common with MLH: location (plasmapause vicinity, no MLT dependence), frequency range $(2-10 \mathrm{kHz})$, frequency width $(1-2 \mathrm{kHz})$, spectral appearance (structure less; banded in frequency; rising tones, possibly whistlers, sometimes embedded). For each case where its frequency range was below $4 \mathrm{kHz}$, this VLF emission was found propagating with a right-hand elliptical polarization, mostly escaping the geomagnetic equator. This survey is based on twenty-seven cases found over 14 months of Cluster data (wave instruments: WHISPER, STAFF and WBD).

To the best of our knowledge, our survey shows, for the first time, that the central frequency value of the MLH is influenced by the geomagnetic activity: the higher the geomagnetic activity, the higher the central frequency of the MLH. Two possible explanations have been suggested. The first one assumes that the waves are generated within a given $f / f_{c e}$ range, near the geomagnetic equator, in the vicinity of the plasmapause. Keeping in mind the $1 / L^{3}$ dependence of $f_{c e}$, the central frequency of the MLH would geomagnetically drift as observed. Another possible explanation would be to consider a moving source located within the plasmapause; the frequency drift would then be explained by a Doppler shift between the moving source and the satellite.

Further studies are foreseen on these mid-latitude hiss emissions, using multi-point and multi-missions measurements. Conjunctions with the IMAGE satellite and its EUV imagers are expected to reveal further insight about their source location. A dedicated study is also foreseen on the possible interaction between whistlers and hiss-like noise bands in the plasmapause region, such as MLH. Finally, another study is also foreseen on a possible interaction between MLH and ELF hiss. 


\section{Appendix}

\begin{tabular}{lllll} 
& & & WBD & STAFF \\
\hline 23 & February & 2001 & C3 & \\
28 & March & 2001 & Off & \\
07 & April & 2001 & Off & X \\
12 & April & 2001 & C3 & \\
24 & April & 2001 & C3 & \\
08 & May & 2001 & C1, C3 & \\
15 & May & 2001 & Off & X \\
05 & June & 2001 & C3 & X \\
20 & June & 2001 & C1 & X \\
24 & June & 2001 & Off & \\
11 & July & 2001 & Off & \\
02 & August & 2001 & C1, C3, C4 & \\
13 & August & 2001 & Off & \\
30 & August & 2001 & Off & \\
18 & September & 2001 & Off & \\
07 & October & 2001 & C1, C4 & \\
10 & October & 2001 & Off & \\
17 & October & 2001 & Off & X \\
26 & October & 2001 & C3 & \\
31 & October & 2001 & Off & \\
10 & November & 2001 & Off & X \\
12 & November & 2001 & Off & X \\
10 & December & 2001 & Off & \\
06 & January & 2002 & Off & X \\
15 & January & 2002 & C1, C4 & \\
08 & February & 2002 & C1, C2, C3, C4 & \\
11 & March & 2002 & C1, C2, C3, C4 & \\
\hline & & & &
\end{tabular}

Acknowledgements. A. Masson deeply thanks K. P. Wenzel, C. P. Escoubet, H. Laakso, R. Grard, J. P. Lebreton, and F. Moser for their support and interest in this work.

Topical Editor T. Pulkkinen thanks two referees for their help in evaluating this paper.

\section{References}

Burgess, W. C. and Inan, U. S.: The role of ducted whistlers in the precipitation loss and equilibrium flux of radiation belt electrons, J. Geophys. Res., 98, 15 643-15 665, 1993.

Carpenter, D. L.: Whistler Evidence of a 'knee' in the magnetospheric ionization density profile, J. Geophys. Res., 68, 1675, 1963.

Carpenter, D. L.: Whistlers and VLF noises propagating just outside the plasmapause, J. Geophys. Res., 83, 45-57, 1978.

Carpenter, D. L. and Anderson, R. R.: An ISEE/Whistler model of equatorial electron density in the magnetosphere, J. Geophys. Res., 97, 1097-1108, 1992.

Carpenter, D. L., Giles, B. L., Chappell, C. R., Décréau, P. M. E., Anderson, R. R., Persoon, A. M., Smith, A. J., Corcuff, Y., and Canu, P.: Plasmasphere dynamics in the duskside bulge region: a new look at an old topic, J. Geophys. Res., 98, 19243-19271, 1993.

Cornilleau-Wehrlin, N., Chauveau, P., Louis, S. et al.: The Cluster Spatio-Temporal Analysis of Field Fluctuations (STAFF) experiment, Space Sci. Rev., 79, 107-136, 1997.
Décréau, P. M. E., Fergeau, D, Krasnosels'kikh, V., et al.: A resonance sounder and wave analyser: performances and perspectives for the Cluster mission, Space Sci. Rev., 79, 157-193, 1997.

Dowden, R. L.: Distinction between mid-latitude VLF hiss and discrete emissions, Planet. Space Sci., 19, 374-376, 1971.

Escoubet, C. P., Russell, C. T., and Schmidt, R.: The Cluster and Phoenix missions, Dordrecht, Kluwer, 1997a.

Escoubet, C. P., Schmidt, R., and Goldstein, M. L.: Cluster - science and mission overview, Space Sci. Rev., 79, 11-32, 1997b.

Gail, W. B. and Carpenter, D. L.: Whistler induced suppression of VLF noise, J. Geophys. Res., 89, 1015-1022, 1984.

Gurnett, D. A., Huff, R. L., and Kirchner, D. L.: The wide-band plasma wave investigation, Space Sci. Rev., 79, 195-208, 1997.

Hayakawa, M. and Sazhin, S. S.: Mid-latitude and plasmaspheric hiss: a review, Planet. Space Sci., 40, 10, 1325-1338, 1992.

Helliwell, R. A.: Whistlers and related ionospheric phenomena, Stanford University Press, Stanford, Calif., 349, 1965.

Helliwell, R. A.: VLF wave stimulation experiments in the magnetosphere from Siple Station, Antarctica, Rev. Geophys., 26, 551-578, 1988.

Helliwell, R. A. and Mende, S. B.: Correlations between $\lambda 4278$ optical emissions and VLF wave events observed at $\mathrm{L} \sim 4$ in the Antarctic, J. Geophys. Res., 85, 3376-3386, 1980.

Helliwell, R. A., Katsufrakis, J. P., and Trimpi, M.: Whistlerinduced amplitude perturbation in VLF propagation, J. Geophys. Res., 78, 4679-4688, 1973.

Higel, B. and Wu, L.: Electron density and plasmapause characteristics at $6.6 R_{E}$ : a statistical study of the GEOS-2 relaxation sounder data, J. Geophys. Res., 89, 1583-1601, 1984.

Johnstone, A. D., Alsop, C., Burge, S., et al.: Peace: A plasma electron and current experiment, Space Sci. Rev., 79, 351-398, 1997.

Lemaire, J. F. and Gringauz, K. I.: The Earth's plasmasphere, Cambridge University Press, Cambridge UK, New York, 1998.

Morgan, M. G.: Equatorial hiss in the topside ionosphere, J. Geophys. Res., 84, 7279-7287, 1979.

Moullard, O., Masson, A., Laakso, H., Parrot, M., Décréau, P., Santolík, O., and André, M.: Density modulated whistler mode emissions observed near the plasmapause, Geophys. Res. Lett., 29(20), 1975, doi:10.1029/2002GL015101, 2002.

Ondoh, T.: Narrow-band plasmapause hiss observed by ISIS satellites, Radio Sci., 28, 629-642, 1993.

Ondoh, T., Nakamura, Y., Watanabe, S., and Murakami, T. Narrow-band VLF hiss observed in the vicinity of the plasmapause, J. Radio Res. Labs, 27, 131, 1980.

Ondoh, T., Nakamura, Y., Watanabe, S., and Murakami, T.: Narrow-band $5 \mathrm{kHz}$ hiss observed in the vicinity of the plasmapause, Planet. Space Sci., 29, 65, 1981.

Paschmann, G., Haaland, S., and Treumann, R.: Auroral plasma physics, Space Sciences Series of ISSI, Dordrecht, Kluwer, 2003.

Rosenberg, T. J., Helliwell, R. A., and Katsufrakis, J. P.: Electron precipitation associated with discrete very-low frequency emissions, J. Geophys. Res., 76, 8445-8452, 1971.

Russell, C. T., Holzer, R. E., and Smith, E. J.: OGO 3 observations of ELF noise in the magnetosphere, The nature of the equatorial noise, J. Geophys. Res., 73, 755-768, 1970.

Santolík, O. and Parrot, M.: Application of wave distribution function methods to an ELF hiss event at high latitudes, J. Geophys. Res., 105, 18 885-18 894, 2000.

Santolík, O., Lefeuvre, F., Parrot, M., and Rauch, J. L.: Complete wave-vector directions of electromagnetic emissions: Application to INTERBALL-2 measurements in the nightside auroral 
zone, J. Geophys. Res., 106, 13 191-13 202, 2001.

Santolík, O., Parrot, M., and Lefeuvre, F.: Singular value decomposition methods for wave propagation analysis, Radio Sci., 38 (1), 1010, 2003.

Santolík, O., Nímec, F., Gereová, K., Macúšová, E., De Conchy, Y., and Cornilleau-Wehrlin, N.: Systematic analysis of equatorial noise below the lower hybrid frequency, Ann. Geophys., 22, 7, 2587-2595, 2004.
Sazhin, S.: Whistler-mode waves in a hot plasma, Cambridge University Press, 1993.

Sonwalkar, V. S.: Magnetospheric LF-, VF-, and ELF-Waves, in Handbook of Atmospheric Electrodynamics, Volume II, edited by Volland, H., 408, CRC press, 1995.

Stix, T. H.: Waves in plasmas, AIP, 1992. 